\title{
Ficolin-2 triggers antitumor and anti-pathogen effects by activating antigen presenting cells and $\mathrm{CD} 8^{+} \mathrm{T}$ cells
}

\author{
Quanquan Ding, Min Liu, Xiao-Lian Zhang*
}

State Key Laboratory of Virology and Medical Research Institute, Hubei Province Key Laboratory of Allergy and Immunology and Department of Immunology Wuhan University School of Basic Medical Sciences, Wuhan 430071, P. R. China.

\section{Article Info}

\section{Article Notes}

Received: October 25, 2017

Accepted: December 04, 2017

\section{${ }^{*}$ Correspondence:}

Dr. Xiao-Lian Zhang,

State Key Laboratory of Virology and Medical Research Institute, Hubei Province Key Laboratory of Allergy and Immunology and Department of Immunology, Wuhan University School of Basic Medical Sciences, Wuhan 430071, P. R. China, E-mail: zhangxiaolian@whu.edu.cn

C 2017 Zhang XL. This article is distributed under the terms of the Creative Commons Attribution 4.0 International License.

\section{Keywords:}

Ficolin-2

Ficolin-A

Anti-tumor

Anti-pathogen

CD8+T

Macrophage

\section{ABSTRACT}

Ficolins are important serum complement lectins with subunits consisting of both collagen-like long thin stretches and fibrinogen-like globular domains with lectin activity usually binding $\mathrm{N}$-acetylglucosamine (GIcNAc). Like collectins such as mannan-binding lectin, ficolins are secreted lectin-type pattern recognition receptors, and activate the lectin pathway of complement activation 1 . Three human ficolins, namely, L-ficolin/P35 (FCN-2/ficolin-2), M-ficolin (FCN-1/ficolin-1), and H-ficolin/Hakata antigen (FCN-3/ficolin-3), and two mouse ficolins (ficolins-A and $-B$ ) have been identified ${ }^{2}-3$. Mouse ficolin-A is closely related to human ficolin-2. They are mainly synthesized in the liver and then secreted into blood circulation.

\section{Ficolin-2 binds and blocks pathogen infection}

Increasing studies have shown that ficolin-2 can bind to glycoproteins of pathogens including viral (Hepatitis C Virus, Influenza A virus and Human Immunodeficiency Virus) and virulent Mycobacterium tuberculosis (M.tb) and inhibit viral and bacterial infections $^{4-10}$ (Figure 1). Ficolin-2 could bind to mannose-capped lipoarabinomannan (ManLAM) of M.tb or Mtb H37Rv and trigger the innate immune response of macrophages for bacteria clearance ${ }^{8}$.

Furthermore, abnormal ficolin-2 expression plays a crucial role in various infectious diseases ${ }^{7-11}$. Ficolin- 2 could bind to envelope glycoprotein E1 and E2 and lead to lectin complement pathway activation $^{11}$, and inhibit Hepatitis C Virus (HCV) early infection ${ }^{5}$. Apolipoprotein E3 (ApoE3) blocks ficolin-2 binding to HCV envelope glycoproteins, enhances HCV infection and mediates viral immune escape $^{5}$

Ficolin-2 binds tumor cells, macrophages and $\mathrm{CD}^{+} \mathrm{T}$ cells in vitro

A recent report showed that ficolin-3 directly attacked cancer cells via a novel complement pathway ${ }^{12}$. Lower ficolin-2 protein expression was found in the tissue samples of Hepatocellular Carcinoma (HCC) patients with HCC metastasis compared to nonmetastasis patients ${ }^{13}$. Despite this, little is known about ficolin-2's functions during malignancy. Recently, Prof. Zhang's research group reported that ficolin-2 could trigger antitumor effect by activating macrophages and $\mathrm{CD}^{+} \mathrm{T}$ cells ${ }^{14}$, in which they revealed the immune roles of serum ficolin-2 in association with immune cells in several 
important cancers, including colon cancer, lung cancer and hepatocarcinoma.

In this work, tumor cells and immune cells were incubated with different concentrations of recombinant ficolin-2 protein and the binding abilities were detected by flow cytometry (FCM) analysis. Firstly, Ding, et al found that ficolin-2 protein could bind to CT26 colon carcinoma cells and lewis lung carcinoma cells, macrophages, dendritic cells (DCs) and CD8 ${ }^{+} \mathrm{T}$ cells, but failed to bind to $\mathrm{CD}^{+} \mathrm{T}$ cells, in vitro in a dose-dependent manner. Both GlcNAc and Mannan, especially GlcNAc, competitively blocked their binding. Among all tested cell types, ficolin- 2 bound to macrophages best and the fibrinogen like domain might be the main region governing the binding of ficolin- 2 to macrophages.

The serum ficolin-2 concentrations in 346 cancer patients and 143 healthy donors were detected by enzymelinked immuno sorbent assay (ELISA) ${ }^{14}$. Much lower levels of serum ficolin-2 were observed in cancer patients compared to healthy donors, these data suggest that low levels of serum ficolin-2 may be related to malignancy.

Ficolin-2 triggers antitumor effect in vivo by activating antigen presenting cells and then $\mathrm{CD}^{+} \mathrm{T}$ cells

Ding et al further determined the roles of ficolin-2/A in the development of tumors in vivo, and observed that significant inhibition of CT26 tumor development in ficolin-2 and ficolin-A-treated mice compared to empty vector treated mice. Similarly, ficolin-2 and ficolin-A treatment also led to a blunted growth of Lewis tumor cells compared to empty vector treated mice. All these data strongly demonstrated that ficolin- 2 and ficolin-A could remarkably inhibit tumor cells growth in vivo.

Further, nude mice were insensitive to treatment with the ficolin-2/ficolin-A expression plasmids, which suggest that $\mathrm{T}$ cells are involved in the antitumor effects triggered by ficolin-2. When early antigen-processing cells (APCs) and $\mathrm{CD}^{+} \mathrm{T}$ cells were depleted, mice exhibited no significant differences in tumor growth between the ficolin2 -treated group and non-ficolin-2-treated group. Ficolin-2 still exhibited antitumor effects when $\mathrm{CD}^{+} \mathrm{T}$ cells and latestage APCs were depleted. These data suggest that earlystage APCs and $\mathrm{CD}^{+} \mathrm{T}$ cells are essential for ficolin- 2 to exert antitumor functions in vivo. In addition, both ficolin-2 and ficolin-A treatments facilitated the recruitment of inducible nitric oxide synthase (iNOS) ${ }^{+} \mathrm{M} 1$ macrophages $\left(\mathrm{iNOS}^{+} \mathrm{F} 4 / 80^{+}\right.$) and $\mathrm{CD}^{+}{ }^{+} \mathrm{CD}^{+}{ }^{+} \mathrm{T}$ cells to tumor sites.

Further research showed that ficolin-2 tilted the balance from M0 or M2 macrophages to M1 macrophages and enhanced macrophage M1-biased polarization response in vitro $^{14}$. Ficolin-2 also enhanced secretion of inflammatory cytokines interleukin-6 (IL-6), tumour necrosis factor-a
(TNF- $\alpha$ ), interferon-gamma (IFN- $\gamma$ ), IL-17A IL-22 and nitric oxide (NO) from macrophages in vitro. Transwell experiments showed that IL- 6 and TNF- $\alpha$ released from macrophages stimulated by ficolin- 2 played major roles in ficolin-2's antitumor effects.

Ficolin-2 can't activate CD8 ${ }^{+} \mathrm{T}$ cells directly ${ }^{14}$. However, mixed lymphocytes reaction (MLR) experiments showed that ficolin-2-activated APCs (macrophages and DCs) enhanced the expression of CD107a, intracellular granzyme $\mathrm{B}(\mathrm{GrB}) /$ perforin and Ki67 of $\mathrm{CD}^{+} \mathrm{T}$ cells and facilitated enhanced antigen-specific cytotoxicity and proliferation of $\mathrm{CD}^{+} \mathrm{T}$ cells. Further, significantly increased expressions of surface CD80, CD86, MHC II and intracellular IL-12 of DCs were observed after the stimulation of ficolin-2. These results suggest that ficolin-2 promotes the maturation of DCs and then activates CD8 ${ }^{+} \mathrm{T}$ cells.

Ficolin-2 exerts antitumor effects depending on Tolllike receptor (TLR) 4 of macrophage

TLR4 was demonstrated to be a receptor of ficolin- $2^{14}$. Flow cytometry analysis showed that ficolin-2 bound to $\mathrm{TLR}^{+/+}$macrophages/DCs in a dose-dependent manner, and the binding ability was much higher than that for TLR4 $\%$ macrophages/DCs. Anti-TLR4 and anti-ficolin-2 antibodies significantly blocked the binding of ficolin-2 to $\mathrm{TLR}^{+/+}$macrophages. Physical associations between ficolin-2 and TLR4 were determined by GST-pull down assay. CD80, CD86, MHC II and IL-12 expression levels in ficolin-2-treated TLR4 ${ }^{+/+}$DCs were significantly higher than those in ficolin-2-treated TLR4 $\%$ DCs. These results suggest that ficolin- 2 promotes the maturation and the antigen presenting ability of DCs via TLR4. And Ficolin-2 induced macrophage activation and M1 polarization response also depended on TLR4, because a significant impairment in the mRNA expressions of inflammatory cytokines IL-6, TNF- $\alpha$ and IFN- $\gamma$, especially TNF- $\alpha$ were observed in the TLR4 $\%$ macrophages compared to those in the $\mathrm{TLR}^{+/+}$ macrophages. The mRNA levels of chemokine ligand (CCL) CCL4 and CCL5 of macrophages, especially CCL5, were also impaired by the deficiency of TLR4 after the stimulation of ficolin-2. Additionally, the mRNA levels of IL-1 $\beta$, IL12 p40 and iNOS in ficolin-2-treated TLR4 $\%$ bone marrow derived M0 macrophage (M0-BMDM) or M2-BMDM were significantly lower than those in ficolin-2-treated TLR4 ${ }^{+/+}$ group. Transforming growth factor (TGF)- $\beta$ and arginase 1 (Arg-1) mRNA levels in ficolin-2-treated TLR4\% M2-BMDM were also higher than those in ficolin-2-treated $\mathrm{TLR}^{+/+}$ M2-BMDM. Ficolin-2 induces M1 polarization response dependent on TLR4.

Ficolin-2 exerts antitumor effects depending on TLR4 of macrophage in vivo. The Hca-f tumor sizes in $\mathrm{TLR}^{+/+}$ mice were significantly smaller than those in TLR $4 \%$ mice at different time course. The Hca-f mouse liver tumor sizes 


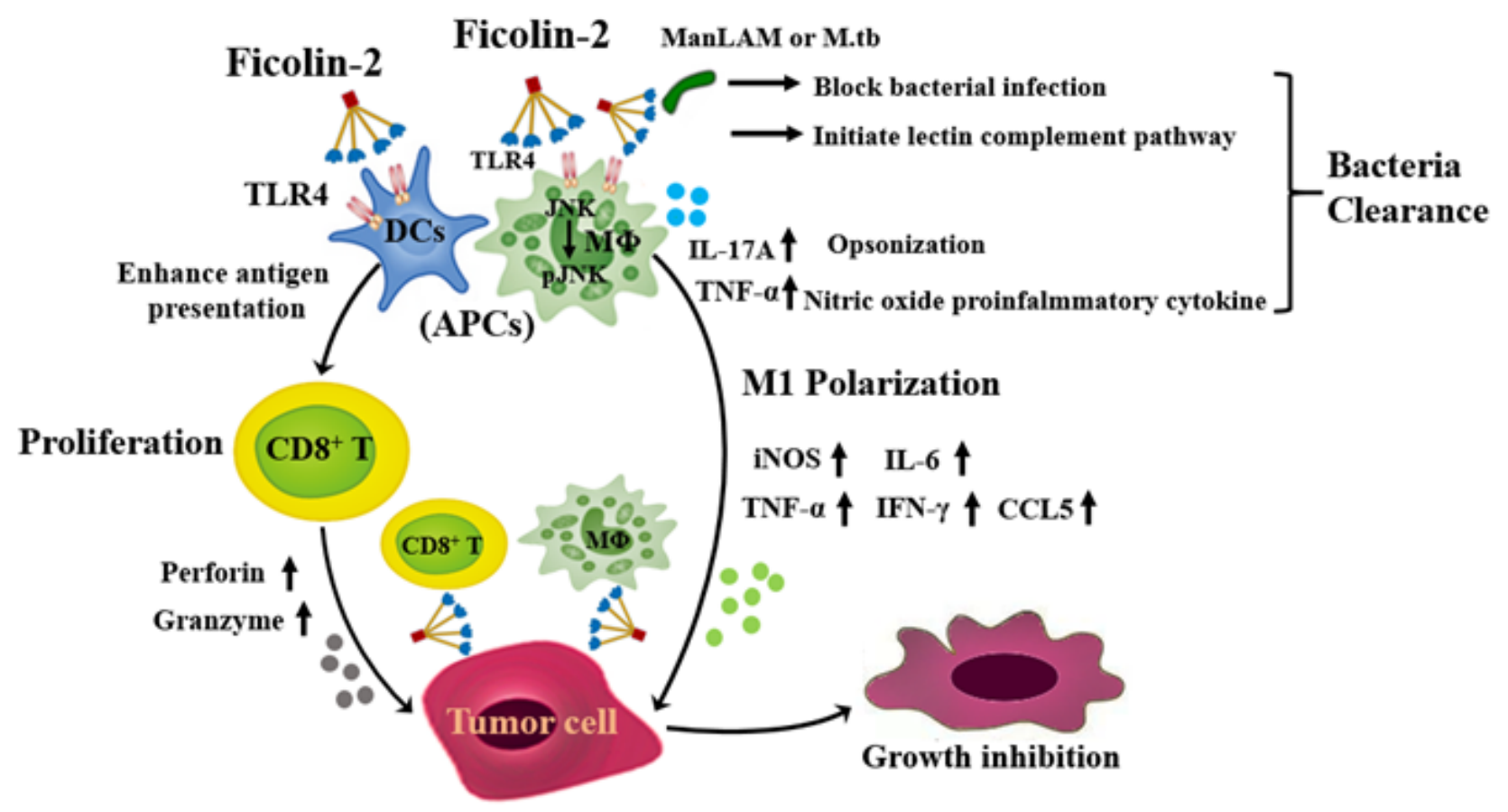

Figure 1. A putative model of immune-mediated anti-pathogen and anti-tumor effects by ficolin-2.

in the empty vector treated $\mathrm{TLR}^{+/+}$mice group were the biggest compared to those in ficolin-2 treated-TLR4 ${ }^{+/+} /$ TLR $4 \%$ mice. These data strongly demonstrate that ficolin-2 significantly inhibit multiple mouse tumor cells growth in different background mice, and TLR4 is involved in macrophage-mediated antitumor effect exerted by ficolin- 2 .

A putative model of immune-mediated anti-tumor effect by ficolin-2 was proposed (Figure 1). The model shows that ficolin-2 binds to TLR4 of macrophage and DCs, induces early macrophages to release proinflammatory cytokines (e.g., TNF-a and IL-6), chemokines (CCL5) and NO; activates M1 macrophage (iNOS ${ }^{+}$) polarization; enhances the ability of APCs (macrophage and DCs) to present antigens to $\mathrm{CD}^{+} \mathrm{T}$ cells; and then enhances antigen-specific $\mathrm{CD}^{+} \mathrm{T}$ cell activation and subsequently protects against tumor progression in vivo. In addition, ficolin-2 acts as a link between tumor cells and immune cells.

Taken together, these new findings may contribute to the development of ficolin- 2 as a novel immunotherapeutic agent that can prevent several important diseases including cancers and infectious diseases.

\section{Acknowledgements}

This work was supported by grants from the National Grand Program on Key Infectious Disease of China (2017ZX10201301), the National Natural Science Foundation of China (31370197, 21572173, 81501377, 81471910, and 91740120), Natural Science Foundation Project and Technological Innovation Major Project of
Hubei Province (2016CFA062, 2016ACA150), the Hubei Province's Outstanding Medical Academic Leader Program (523-276003), the Science and Technology Program of Wuhan (201150530141) and the Wuhan Applied Basic Research Project (2015060101010030).

\section{References}

1. Matsushita M. Ficolins: Complement-Activating Lectins Involved in Innate Immunity. Journal of Innate Immunity. 2010; 2: 24-32.

2. Lu J, Le Y. Ficolins and the fibrinogen-like domain. Immunobiology. 1998; 199: 190-199.

3. Endo Y, Liu Y, Kanno K, et al. Identification of the mouse H-ficolin gene as a pseudogene and orthology between mouse ficolins $\mathrm{A} / \mathrm{B}$ and human L-/M-ficolins. Genomics. 2004; 84: 737-744.

4. Pan $\mathrm{Q}$, Chen $\mathrm{H}$, Wang $\mathrm{F}$, et al. L-ficolin binds to the glycoproteins hemagglutinin and neuraminidase and inhibits influenza A virus infection both in vitro and in vivo. Journal of Innate Immunity. 2012; 4: 312-324.

5. Zhao Y, Ren Y, Zhang X, et al. Ficolin-2 inhibits hepatitis $\mathrm{C}$ virus infection, whereas apolipoprotein E3 mediates viral immune escape. Journal of immunology. 2014; 193: 783-796.

6. Aoyagi Y, Adderson EE, Min JG, et al. Role of L-ficolin/mannosebinding lectin-associated serine protease complexes in the opsonophagocytosis of type III group B streptococci. Journal of immunology. 2005; 174: 418-425.

7. $\mathrm{Hu} \mathrm{YL}$, Luo FL, Fu JL, et al. Early increased ficolin-2 concentrations are associated with severity of liver inflammation and efficacy of antiviral therapy in chronic hepatitis C patients. Scand J Immunol. 2013; 77: $144-150$.

8. Luo F, Sun X, Wang Y, et al. Ficolin-2 defends against virulent Mycobacteria tuberculosis infection in vivo, and its insufficiency is associated with infection in humans. PloS one. 2013; 8: e73859.

9. Chen $\mathrm{T}, \mathrm{Hu} \mathrm{Y}$, Ding $\mathrm{Q}$, et al. Serum ficolin-2 concentrations are 
significantly changed in patients with hepatitis B virus infection and liver diseases. Virologica Sinica. 2015; 30: 249-260.

10. Luo F, Chen T, Liu J, et al. Ficolin-2 binds to HIV-1 gp120 and blocks viral infection. Virologica Sinica. 2016; 31: 406-414.

11. Liu J, Ali MA, Shi Y, et al. Specifically binding of L-ficolin to N-glycans of HCV envelope glycoproteins E1 and E2 leads to complement activation. Cellular \& molecular immunology. 2009; 6: 235-244.

12. Lei X, Liu C, Azadzoi K, et al. A novel IgM-H-ficolin complement pathway to attack allogenic cancer cells in vitro. Sci Rep. 2015; 5: 7824.

13. Yang G, Liang Y, Zheng T, et al. FCN2 inhibits epithelial-mesenchymal transition-induced metastasis of hepatocellular carcinoma via TGFbeta/Smad signaling. Cancer letters. 2016; 378: 80-86.

14. Ding $Q$, Shen Y, Li D, et al. Ficolin-2 triggers antitumor effect by activating macrophages and CD8+ T cells. Clin Immunol. 2017; 183: 145-157. 